\title{
Distributed Power Balance and Damping Control for High Power Multiport LCL DC Hub
}

\author{
Weixing Lin, Dragan Jovcic*, Seyed Mahdi Fazeli \\ School of Engineering, King's College, University of Aberdeen, Aberdeen AB24 3UE, UK
}

Abstract - DC hubs are centrally located in DC grids and they are expected to operate autonomously under wide range of operating conditions. To achieve secure operation without intervention from dispatcher and to damp the internal oscillatory modes, a distributed power balance and damping controller for high power multiport LCL (Inductor-Capacitor-Inductor) DC hub is proposed in this paper. Natural power balance mechanism of the hub is analyzed. To avoid over-voltage and over-power during un-balanced power orders, an actively controlled distributed power balance control for each port of the hub is proposed. As LCL circuit is used in the hub, there might exist prolonged oscillations in the hub. Small signal modelling and eigenvalue studies are used to identify the dominant oscillation mode of the hub. The participation factors are calculated to identify the state variables that best represent the dominant oscillation modes. The residue analysis is used to pair the feedback signals and design the structure of the damping controller. Robustness of the damping loop against topology changes is analyzed. Effectiveness of the designed controller in terms of secure operation and transient responses is verified by extensive PSCAD/EMTDC simulations on a 4-port test LCL DC hub.

Key Words - DC power systems; LCL DC Hub; DC-DC power conversion; HVDC transmission; Damping control; Residue Analysis

*Corresponding author. Tel: +44 Tel.: +44 1224272 336; fax: +44 1224272497.

E-main addresses:(weixinglin@abdn.ac.uk, *d.jovcic@abdn.ac.uk, seyedmahdifazeli@abdn.ac.uk). 


\section{Introduction}

With the development of renewable energy and voltage source converter (especially modular multilevel converter), there has been increasing interest in building multi-terminal HVDC systems and DC grids [1]-[4]. DC grids can be built by interconnecting different DC transmission lines using DC circuit breakers (CB) [5], but such topology confronts the challenge of coordination of protection for a large DC grid [5]-[8]. Because of very small DC cable impedance, the DC faults will cause widespread voltage collapse and require fast (few $m s$ ) but discriminative (coordinated) protection action. It will be very challenging to achieve high DC grid reliability if DC CBs are used solely as protection means.

DC-DC converters [6] can be used to interconnect two dc transmission lines of the same or different dc voltages and they reliably stop propagation of a DC faults.

The multiport LCL DC hub [7]-[8] is another approach in building DC grids which provides cost-effective solution to interconnect more than 2 DC lines and it achieves reliable DC fault protection. DC hub becomes electronic substation which achieves voltage stepping, power flow control and DC fault isolation, and the study in [7] focuses on the design of such LCL hub. The study in [8] investigates methods of improving reliability and redundancy of the hub by introducing multi-phase, multi-module and redundant phase concept. The studies in [7] and [8] however use open loop or rudimentary DC hub control.

As the DC grid terminals and LCL DC hub will be placed in remote areas such as the North Sea, issues arise as how to autonomously balance the power in a DC grid and in the DC hub. There have been extensive researches regarding power balancing in a VSC-HVDC and multi-terminal HVDC system [9]-[13].

Three different communication-independent strategies, the frequency modulation method, the frequency modulation together with reduction of $\mathrm{AC}$ voltage and the reduction of $\mathrm{AC}$ voltage with a 
newly proposed voltage-dependent current modulation strategy were studied in [9] to achieve frequency response and fault ride through in an offshore wind farm connected to AC grid through VSC-HVDC. A coordinated direct current matching control strategy based on wide-area supervisory control and data acquisition system was proposed in [10] to achieve coordinated DC current control between offshore wind power terminals and onshore VSC converters in a Multiterminal DC (MTDC) system. Different methods for DC node voltage control for HVDC grids were summarized in [11]. The droop control is used to achieve power balance in a HVDC grid.

Hierarchical power control of MTDC grids is proposed in [12], wherein the DC voltage droop control is used as the primary control to ensure power balance of the DC grid. A secondary control is used to adjust the voltage reference of the droop control to track the power reference. A further tertiary control is located at central DC re-dispatcher to achieve more optimal operation. Similar as [12], reference [13] uses droop control to ensure stability and optimal power flow to set reference values for droop control.

Because of the fast and decoupled active power and reactive power control capability of VSC converters, there have also been extensive studies on damping power oscillations in a MTDC using VSC converters [14]. MTDC grid control can also contribute damping of inter-area oscillations in large AC systems [15].

However DC hub brings unique control challenges, because of new DC grid topology, because of central role in power flow and because of complex internal dynamics. The LCL DC hub functions as the summing station that enables power trading between different transmission system operators (TSO). Each TSO will send the power exchange order to the particular port in the hub at their wishes, while the sum of all power orders received in the hub may not necessarily sum to zero. The primary challenge with hub controller design is how to deliver power at each port as close as possible to the desired value while maintaining instantaneous power balance and stability in the hub. There will not 
be energy storage within the hub and therefore fast internal power balance is essential. All ports in a hub should have the same control topology, considering that any number of ports can be connected/disconnected at any time. It is essential that hub continues operation when a DC fault requires tripping a local port.

An initial topology for a distributed DC hub controller for a simple 6-port DC hub is presented in [16]. The current paper advances further the control system from [16], with the aim to offer in depth control design study, analytical stability assessment, damping of oscillatory modes and evaluation of a generic DC hub controller.

As LCL circuit is used inside the hub, there exists natural LCL resonance, which cannot be eliminated in the main circuit design stage, and which may cause prolonged oscillations in the transient response. The impact of LCL resonance was not observed in [16] since LCL resonant frequency was far away from the operating frequency for the particular test system in [16]. This paper will study a distributed damping controller for the LCL DC hub, in order to provide adequate transient responses for wide range of hub applications and all operating conditions.

\section{Topology and basic circuit equations of the LCL Dc Hub}

\subsection{Topology}

Structure of a 4-port LCL DC hub is shown in Fig.1 where a 3-phase topology is adopted [7]. A general DC hub comprises $N$ ports which connect to respective DC sources and which in an actual hub would be DC lines or DC networks. Each of the $N$ ports is comprised of 3 inductors $L_{\mathrm{i}}, 3$ capacitors $\mathrm{C}_{\mathrm{i}}$ and one DC/AC bridge with switches $\left(S_{1_{-} \mathrm{i}}-S_{6_{-}}\right)$. The inner AC circuit breakers $(\mathrm{CB})$ enable isolation of the ports. Note that the central capacitor is distributed between the ports to enable proper reactive power balance when ports are tripped. The inner LCL circuit is designed for power exchange between different ports and fault current limiting [7]. 


\subsection{Basic Circuit Equations}

In a $d q$ frame rotating at the operating frequency of the hub, steady state equation for the current of each port $i$ is

$$
\begin{aligned}
I_{i d}+j I_{i q} & =\frac{\left(M_{i d} V_{i r}+j M_{i q} V_{i r}\right)-\left(V_{c d}+j V_{c q}\right)}{j \omega_{o} L_{i}} \\
& =\frac{M_{i q} V_{i r}-V_{c q}}{\omega_{o} L_{i}}+j \frac{V_{c d}-M_{i d} V_{i r}}{\omega_{o} L_{i}}
\end{aligned}
$$

Where $M_{\mathrm{id}}$ and $M_{\mathrm{iq}}$ are $d$-channel and $q$-channel modulation indices (control signals) of each port, $V_{\mathrm{cd}}$ and $V_{\mathrm{cq}}$ are the $d$-channel and $q$-channel components of the capacitor voltage $v_{\mathrm{c}}$ when projected to the rotating $d q$ frame. $V_{\text {ir }}$ is the rated RMS magnitude of the phase voltage $v_{\mathrm{i}}$ of port $i$, which is constant since it is linked with constant DC voltage. $V_{\mathrm{cr}}$ is the rated RMS value of the capacitor voltage $v_{\mathrm{c}}, \omega_{\mathrm{o}}$ is the operating frequency of the inner AC circuit (same for the whole hub).

Each $L_{\mathrm{i}}$ and $C_{\mathrm{i}}$ are designed by [7]:

$$
\begin{gathered}
L_{i}=\frac{V_{i r} \sqrt{V_{c r}^{2}-V_{i r}^{2}}}{\omega_{o} P_{i r}} \\
C=\sum_{i=1}^{N} C_{i}=\frac{1}{\omega_{o} V_{c r}^{2}} \sum_{i=1}^{N} \frac{P_{i r} \sqrt{V_{c r}^{2}-V_{i r}^{2}}}{V_{i r}}
\end{gathered}
$$

Active power per phase of a port $i$ is:

$$
P_{i}=M_{i d} V_{i r} I_{i d}+M_{i q} V_{i r} I_{i q}=\frac{M_{i q} V_{i r} V_{c d}-M_{i d} V_{i r} V_{c q}}{\omega_{o} L_{i}}
$$

where injecting active power to the common AC bus is defined as the positive power direction.

According to the Kirchhoff's current law (KCL),

$$
\sum_{i=1}^{N} I_{i d}=0, \sum_{i=1}^{N} I_{i q}=0
$$

Where $N$ is the total number of ports. Substitute each $I_{\text {id }}$ and $I_{\text {iq }}$ calculated from (1) into (5), the 
following equations for the common bus AC voltage can be derived.

$$
\begin{array}{r}
K_{c} V_{c d}=\sum_{i=1}^{N} \frac{M_{i d} V_{i r}}{\omega_{o} L_{i}} \\
K_{c} V_{c q}=\sum_{i=1}^{N} \frac{M_{i q} V_{i r}}{\omega_{o} L_{i}} \\
K_{c}=\sum_{i=1}^{N} \frac{V_{i r}^{2}}{V_{c r}^{2} \omega_{o} L_{i}}, \quad K_{c}>0
\end{array}
$$

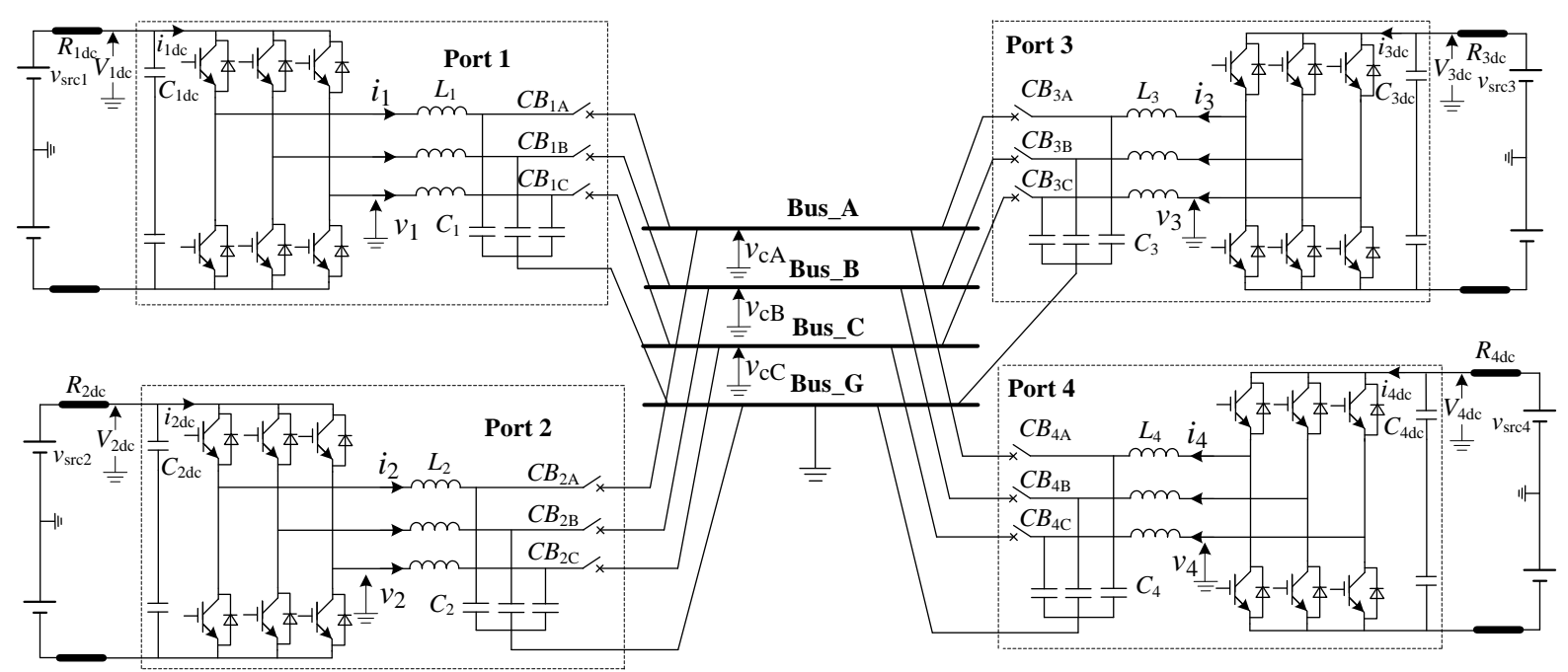

Fig. 1. Three phase 4-port DC hub topology

\section{Distributed power balancing control of the LCL DC hub}

\subsection{Control function of active power and common bus AC voltage}

Each port has its own controller which should deliver reference power to/from the local DC system. However, it is also required to achieve instantaneous power balance in the hub and to keep capacitor voltage within the required limits. If there is power imbalance on the central bus, then capacitor voltage will either collapse or rapidly rise to high values. In order to achieve hub security under all operating conditions and topologies (port tripping), it is proposed to develop a distributed capacitor voltage controller which will be located at each port. 
The goal of the capacitor voltage control is to control $V_{\mathrm{cd}}$ to its rated value $V_{\mathrm{cr}}$ and to control $V_{\mathrm{cq}}$ to zero, namely,

$$
V_{c d}=V_{c r}, V_{c q}=0
$$

Under the conditions of (9), the active power equation of (4) can be simplified as,

$$
P_{i}^{i d e a l}=\frac{M_{i q} V_{i r} V_{c r}}{\omega_{o} L_{i}}
$$

Equation (10) indicates $M_{\mathrm{iq}}$ can be used to control the active power $P_{\mathrm{i}}$, and also that under the proposed control topology the $q$-control channel becomes linear. Since $M_{i q}$ is used to control port " $i$ " power, the challenge is how to accommodate $V_{c q}$ control, as any manipulation of $M_{\text {iq }}$ also directly affects $V_{\text {cq }}$ according to (7).

According to (6) $V_{\text {cd }}$ is proportional to each $M_{\text {id }}$. Therefore, the voltage $V_{\text {cd }}$ can be controlled to $V_{\text {cr }}$ if $M_{\mathrm{id}}$ of each port stays at the rated value, namely,

$$
M_{i d}=M_{i d r}
$$

Where $M_{\text {idr }}$ is the rated value of $M_{\text {id }}$.

\subsection{Inherent power balance mechanism of the hub}

Sum of all the $P_{\mathrm{i}}$ on $N$ ports in (4) equals zero in steady-state, and therefore,

$$
V_{c q}=\frac{\sum_{i=1}^{N} \frac{M_{i q} V_{i r} V_{c d}}{\omega_{o} L_{i}}}{\sum_{i=1}^{N} \frac{M_{i d} V_{i r}}{\omega_{o} L_{i}}}=\frac{\sum_{i=1}^{N} P_{i}^{i d e a l}}{K_{c} V_{c d}}
$$

Throughout this paper, $M_{\text {id }}$ will always be set to be greater than zero while $M_{\text {iq }}$ can have positive or negative values. Equation (12) shows that $V_{\mathrm{cq}}$ is indicator of the power balance in the hub and therefore the chosen control goal of (9) with $V_{c q}=0$ ensures continuous power balance under all conditions. 
We will examine next the situation if $V_{c q}$ is not controlled. If $\sum_{i=1}^{N} P_{i}^{i d e a l}>0$, this indicates that there is more scheduled injecting power than the scheduled absorbing power and we will have $V_{c q}>0$ according to (7). A positive $V_{c q}$ will, according to (4), decreases the power of injecting ports and increase the power of absorbing ports, which is therefore an inherent stabilizing loop. The controllers on ports will try to keep power at reference and they will then increase or decrease their $M_{\mathrm{iq}}$ until $M_{\mathrm{iq}}$ hits limits. It will not be possible to achieve the active power balance in the hub without port overload. Another issue is that as $V_{c q}$ varies, the magnitude of capacitor voltage may reach unacceptable values. In the case of $\sum_{i=1}^{N} P_{i}^{\text {ideal }}<0$, the analysis is similar.

It is therefore necessary to actively regulate $V_{c q}=0$ and this can be achieved using $q$-channel as seen in (7). Distributed control is necessary to ensure adequate security in case of hub/port outages.

\subsection{Distributed Control of Each Port of the hub}

Fig. 2 shows the proposed distributed control structure of the LCL dc hub. Each port of the hub is equipped with the same controller. Since the inner LCL circuit of the LCL DC hub is a passive AC network and all the high frequency capacitors, inductors and CBs are located in one or several nearby buildings, a single voltage controlled oscillator is used to generate global reference angle for all the ports. This method is similar to the case of connecting VSC to a dead AC grid. No PLL will be required.

The port controller is composed of three control loops acting on $M_{i q}$ 'active power loop', 'damping loop' and a ' $V_{\text {cq }}$ loop', and two control loops acting on $M_{i d}$ : ' $V_{\text {cd }}$ loop' and 'damping loop'. The subscript $p u$ and $r e f$ respectively stands for per unit and reference values. To avoid abrupt change of power reference, a rate limiter with default value of $\pm 10 \mathrm{pu} / \mathrm{s}$ is applied at the input of each $P_{\text {iref. }}$. 
The $V_{\text {cq }}$ loop acts as an actively controlled power balance loop with integral control action. The common approach of droop feedback [4][13] is not used since it would lead to steady-state error in $V_{c q}$. Since all ports use PI (proportional-integrator) control, this guarantees that $V_{c q}=0$ even if controls on some ports are saturated. A port saturation can easily occur if local power reference is not achievable under operating conditions.

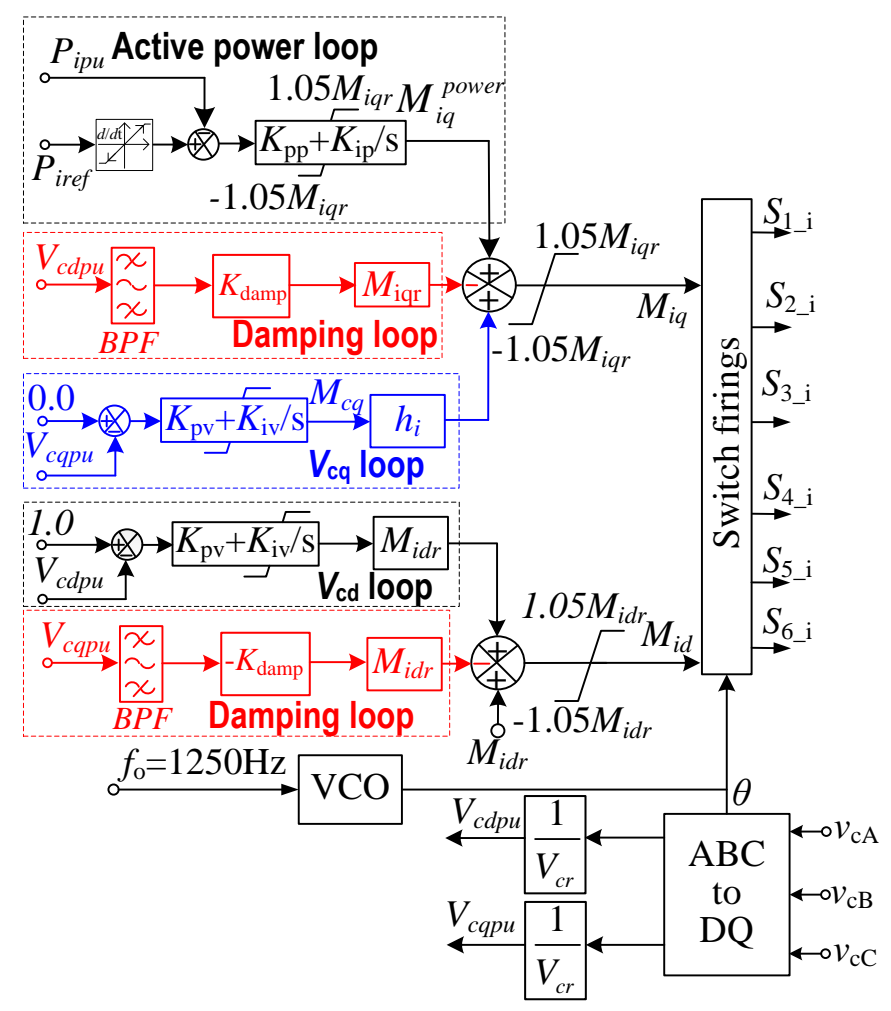

Fig. 2. Distributed control structure of each port

A coefficient $h_{\mathrm{i}}$ is multiplied with $M_{\mathrm{cq}}$ in the $V_{\mathrm{cq}}$ loop to adjust the contribution of a port to the active power balance. According to $(10), h_{\mathrm{i}}$ can be set to $M_{\mathrm{iqr}}$ so that a nonzero $V_{\mathrm{cq}}$ will bring the same percentage of power change to all the injecting ports or all the absorbing ports. The $h_{\mathrm{i}}$ can also be set to zero so that a port $i$ will not participate in the DC hub power balancing (as an example if it connects to a wind farm).

We next analyze the case that power references are unbalanced $\sum_{i=1}^{N} P_{\text {iref }} \neq 0$. Taking $\sum_{i=1}^{N} P_{\text {iref }}<0$ as an 
example, control signals from all absorbing power controllers $M_{i q}^{p o w e r}$ will hit lower limits $-1.05 M_{\text {iqr. }}$. A negative $V_{\mathrm{cq}}$ will then show up according to (12). A positive $M_{\mathrm{cq}}$ will then be produced by the $V_{\mathrm{cq}}$ loop in Fig. 2 which will increase the $M_{\text {iq }}$ of all the absorbing ports (to decrease the absorbed power) and increase $M_{i q}$ of all injecting ports (to increase the injected power). A new balanced operating point will finally be achieved. As $M_{i q}^{\text {power }}$ of the injecting ports do not hit the upper limits, power of the injecting ports will be controlled to their reference values.

\section{LCL resonance and dominant oscillation mode of the LCL circuit}

\subsection{LC resonant frequency of $L C L D C$ hub}

As LCL circuit is used in the hub, there exists LC resonant frequency $\omega_{\mathrm{n}}$, which may cause prolonged oscillations. A particular problem occurs if resonant frequency is close to the operating frequency $\omega_{0}$. Fig. 3 shows the simplified equivalent circuit for one phase of the internal LCL circuit of Fig. 1 where $C$ is the sum of the capacitor on all the ports.

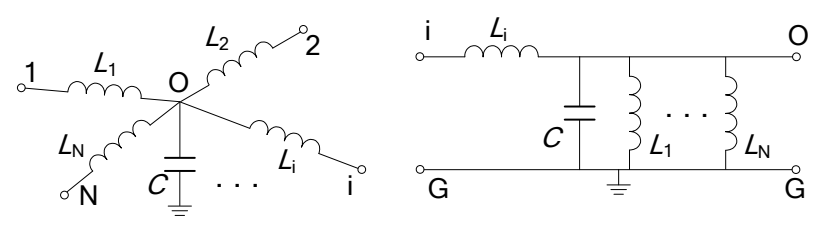

Fig. 3. Simplified equivalent Circuits of $N$ port LCL DC hub

The impedance of the hub viewed from each port is

$$
Z_{i}=\frac{1}{j \omega C+\sum_{k=1, k \neq i}^{N} \frac{1}{j \omega L_{k}}}+j \omega L_{i}
$$

Setting (13) to zero, the natural LCL frequency $\omega_{\mathrm{n}}$ can be calculated as:

$$
\omega_{n}=\sqrt{\frac{1}{C} \sum_{k=1}^{N} \frac{1}{L_{k}}}
$$


If ac voltage at the above frequency is supplied at port $i$, then current at that port will be infinity. It is seen that (14) is identical for all ports and represents global hub resonance. It is also seen in (14) that $\omega_{\mathrm{n}}$ depends only on the circuit parameters. However if some ports are tripped, then $\omega_{\mathrm{n}}$ will change.

\subsection{Relationship of LC resonant frequency with the power rating and voltage rating of each port}

This section explores the relationship of $\omega_{n}$ with the design parameters of the hub. Typically, all the port rated variables are given $\left(P_{i r}, V_{i r}\right)$ and there is only one degree of freedom in the design, which is the selection of $V_{c r}$ [7]. $V_{\text {cr }}$ is typically designed to be $20 \%$ higher than the highest $V_{\text {ir }}[7]$. Substitute (2) and (3) into (14) results,

$$
\omega_{n}=\omega_{o} V_{c r} \sqrt{\frac{1}{\sum_{i=1}^{N} \frac{P_{i r} \sqrt{V_{c r}^{2}-V_{i r}^{2}}}{V_{i r}}} \sum_{k=1}^{N} \frac{P_{i r}}{V_{i r} \sqrt{V_{c r}^{2}-V_{i r}^{2}}}}
$$

Equation (15) is rather complex. To get qualitative relationship between $\omega_{\mathrm{n}}, P_{\mathrm{ir}}, V_{\mathrm{ir}}$ and $V_{\mathrm{cr}}$, a 1 -port hub will be analyzed. In the case of 1-port hub $(N=1)$, equation (15) is simplified as

$$
\omega_{n}=\frac{\omega_{o}}{\sqrt{1-\left(V_{i r} / V_{c r}\right)^{2}}}
$$

We can see from (16) that $\omega_{\mathrm{n}}$ will be close to $\omega_{\mathrm{o}}$ if $V_{\mathrm{cr}}$ is significantly higher than $V_{\text {ir }}$ and $\omega_{\mathrm{n}}$ will be significantly higher than $\omega_{\mathrm{o}}$ if $V_{\mathrm{cr}}$ is close to $V_{\mathrm{ir}}$. An oscillating component with frequency $\omega_{\mathrm{n}}$ close to the operating frequency $\omega_{\mathrm{o}}$ will get easily excited but difficult to damp, while a $\omega_{\mathrm{n}}$ significantly higher than $\omega_{\mathrm{o}}$ can be easily damped by filters.

As an example, the 6-port test hub used in [16] has $V_{\text {cr }}$ close to each $V_{\text {ir }}$ (since DC voltages of all ports are very close) and $f_{\mathrm{n}}$ is $2035 \mathrm{~Hz}$, which is significantly higher than the operating frequency of $1250 \mathrm{~Hz}$. Therefore no un-damped oscillations have been observed. 


\subsection{Small signal analysis of the hub}

Small signal analysis will be used to design damping controller. In a rotating $d q$ frame, dynamic equations of the inductor currents are:

$$
\begin{aligned}
& \frac{d I_{i d}}{d t}=\frac{1}{L_{i}}\left(V_{i d}-V_{c d}+\omega_{o} L_{i} i_{i q}-R_{i} I_{i d}\right)=\frac{1}{L_{i}}\left(k_{m} M_{i d} V_{i d c}-V_{c d}+\omega_{o} L_{i} i_{i q}-R_{i} I_{i d}\right) \\
& \frac{d I_{i q}}{d t}=\frac{1}{L_{i}}\left(V_{i q}-V_{c q}-\omega_{o} L_{i} i_{i d}-R_{i} I_{i q}\right)=\frac{1}{L_{i}}\left(k_{m} M_{i q} V_{i d c}-V_{c q}-\omega_{o} L_{i} i_{i d}-R_{i} I_{i q}\right)
\end{aligned}
$$

State dynamic equations for the capacitor voltage are:

$$
\begin{aligned}
& \frac{d V_{c d}}{d t}=\frac{1}{C}\left(\sum_{i=1}^{N} I_{i d}+\omega_{o} C V_{c q}\right) \\
& \frac{d V_{c q}}{d t}=\frac{1}{C}\left(\sum_{i=1}^{N} I_{i q}-\omega_{o} C V_{c d}\right)
\end{aligned}
$$

The state equation for the DC side is:

$$
\frac{d V_{i d c}}{d t}=\frac{1}{C_{i d c}}\left(\frac{V_{s r c i}-V_{i d c}}{R_{i d c}}-i_{i d c}\right)
$$

Where $V_{\text {srci }}$ is the DC voltage of the DC battery, $V_{\text {idc }}$ is the pole-to-ground DC voltage at DC terminal of a port, $i_{\mathrm{idc}}$ is the DC current flows into the converter bridge, $C_{\mathrm{idc}}$ and $R_{\mathrm{idc}}$ are respectively the DC capacitance and the DC resistor. Measuring points of these signals are shown in Fig. 1.

The $i_{\text {idc }}$ can be calculated by [21]:

$$
i_{i d c}=\frac{M_{p}}{4} k_{m}\left(M_{i d} I_{i d}+M_{i q} I_{i q}\right)
$$

Where $M_{\mathrm{p}}$ is the number of phases. $M_{\mathrm{p}}=3$ for the three-phase hub shown in Fig. $1, k_{\mathrm{m}}$ is a coefficient related to the modulation method, $k_{\mathrm{m}}=4 / \pi$ for the modulation method employed in the hub[7].

Substituting (22) into (21) results:

$$
\frac{d V_{i d c}}{d t}=\frac{1}{C_{i d c}}\left(\frac{V_{s r c i}-V_{i d c}}{R_{i d c}}-i_{i d c}\right)=-\frac{M_{p} k_{m}}{4 C_{i d c}} M_{i d} I_{i d}-\frac{M_{p} k_{m}}{4 C_{i d c}} M_{i q} I_{i q}-\frac{1}{C_{i d c} R_{i d c}} V_{i d c}+\frac{1}{C_{i d c} R_{i d c}} V_{s r c i}
$$


In (17) (18) and (23) the output from the controller $\left(M_{\mathrm{id}}, M_{\mathrm{iq}}\right)$ are multiplied with the state variables which introduces non-linarites. By linearizing the non-linear terms $M_{i d} V_{i d c}$ in (17), $M_{i q} V_{i d c}$ in (18), $M_{i d} I_{i d}$ and $M_{i q} I_{i q}$ in (23), and writing (19) and (20) in deviation form, the equations (17)-(23) can be rewritten as:

$$
\begin{aligned}
& \frac{d \Delta I_{i d}}{d t}=\frac{-R_{i}}{L_{i}} \Delta I_{i d}+\omega_{o} \Delta I_{i q}-\frac{1}{L_{i}} \Delta V_{c d}+\frac{k_{m} M_{i d 0}}{L_{i}} \Delta V_{i d c}+\frac{k_{m} V_{i d c 0}}{L_{i}} \Delta M_{i d} \\
& \frac{d \Delta I_{i q}}{d t}=-\omega_{o} \Delta I_{i d}-\frac{R_{1}}{L_{1}} \Delta I_{i q}-\frac{1}{L_{i}} \Delta V_{c q}+\frac{k_{m} M_{i q 0}}{L_{i}} \Delta V_{i d c}+\frac{k_{m} V_{i d c 0}}{L_{i}} \Delta M_{i q} \\
& \frac{d \Delta V_{c d}}{d t}=\frac{1}{C} \sum_{i=1}^{N} \Delta I_{i d}+\omega_{o} \Delta V_{c q} \\
& \frac{d \Delta V_{c q}}{d t}=\frac{1}{C} \sum_{i=1}^{N} \Delta I_{i q}-\omega_{o} \Delta V_{c d} \\
& \frac{d \Delta V_{i d c}}{d t}=-\frac{M_{p} k_{m}}{4 C_{i d c}} M_{i d 0} \Delta I_{i d}-\frac{M_{p} k_{m}}{4 C_{i d c}} M_{i q 0} \Delta I_{i q}-\frac{1}{C_{i d c} R_{i d c}} \Delta V_{i d c}-\frac{M_{p} k_{m}}{4 C_{i d c}} I_{i d 0} \Delta M_{i d}-\frac{M_{p} k_{m}}{4 C_{i d c}} I_{i q 0} \Delta M_{i q}+\frac{1}{C_{i d c} R_{i d c}} \Delta V_{s r c i}
\end{aligned}
$$

Express (24) to (28) in a matrix form results:

$$
\frac{d}{d t} \Delta \boldsymbol{x}_{e}=\boldsymbol{A}_{e} \Delta \boldsymbol{x}_{e}+\boldsymbol{B}_{e} \Delta \boldsymbol{M}_{d q}
$$

\subsection{Dominant oscillatory mode of the LCL circuit}

A 4-port 3-phase $1080 \mathrm{MW}$ hub operated at $f_{0}=1250 \mathrm{~Hz}$, is used as a test system to study the controller design. Parameters of the test hub are given in Table 1. The ports are purposely selected to have widely different power and voltage ratings to represent difficult operating conditions.

The eigenvalue analysis of the system matrix $\boldsymbol{A}_{\mathrm{e}}$ shows that the dominant oscillatory mode is $-7.1 \pm 680.6 \mathrm{i}$. Damping ratio and frequency of the dominant mode are respectively 0.01 and $108.3 \mathrm{~Hz}$.

The global resonant frequency calculated using (14) is $1358 \mathrm{~Hz}$. Viewed in a $d q$ frame rotating at $1250 \mathrm{~Hz}$, the resonant frequency is $108 \mathrm{~Hz}$. The dominant oscillation frequency calculated from 
eigenvalue study is almost identical as the frequency calculated from(14), which confirms accuracy of the linearized model.

Table 1 Parameters of the 4-port test LCL hub

\begin{tabular}{|l|l|l|l|l|}
\hline Port & 1 & 2 & 3 & 4 \\
\hline$V_{\mathrm{idc}}(k \mathrm{~V})$ & 50 & 100 & 300 & 400 \\
\hline $3 * P_{i r}(\mathrm{MW})$ & 180 & 750 & 900 & 330 \\
\hline$L_{\mathrm{i}}(\mathrm{H})$ & 0.039 & 0.018 & 0.038 & 0.105 \\
\hline$C_{\mathrm{i}}(\mathrm{uF})$ & 0.411 & 0.844 & 0.277 & 0.058 \\
\hline$M_{i d r}$ & 0.094 & 0.188 & 0.564 & 0.752 \\
\hline$M_{i q r}$ & 0.945 & 0.931 & 0.764 & 0.580 \\
\hline Initial Power Direction & -1 & 1 & -1 & 1 \\
\hline$V_{\text {cr }}(k \mathrm{~V})$ & & & & \\
\hline
\end{tabular}

\section{Design of the Damping Controller}

\subsection{Participation factor of the dominant oscillatory mode}

The participation factors [17]-[18] are used to select the best states as feedback signals for damping of dominant oscillatory mode. Participation factors are computed by

$$
P=\varphi_{i} \otimes \psi_{i}
$$

Where $\varphi_{\mathrm{i}}$ is the right eigenvector of matrix $A_{e}$ in (29) associated with the eigenvalue $\lambda_{\mathrm{i}}, \psi_{\mathrm{i}}$ is the left eigenvector. $\otimes$ denotes element-by-element multiplication.

Table 2 shows the participation factors with respect to the dominant oscillatory mode $-7.1 \pm 680.6 \mathrm{i}$. As can be seen, the participation factors related to $V_{c d}$ and $V_{c q}$ are equal and at maximum values. 
Table 2 Participation factors for different states (normalized based on the participation factor of $V_{\mathrm{cd}}$ )

\begin{tabular}{llllllllllllll}
\hline$I_{1 \mathrm{~d}}$ & $I_{1 \mathrm{q}}$ & $I_{2 \mathrm{~d}}$ & $I_{2 \mathrm{q}}$ & $I_{3 \mathrm{~d}}$ & $I_{3 \mathrm{q}}$ & $I_{4 \mathrm{~d}}$ & $I_{4 \mathrm{q}}$ & $V_{\mathrm{cd}}$ & $V_{\mathrm{cq}}$ & $V_{1 \mathrm{dc}}$ & $V_{2 \mathrm{dc}}$ & $V_{3 \mathrm{dc}}$ & $V_{4 \mathrm{dc}}$
\end{tabular}

$\begin{array}{llllllllllllll}0.22 & 0.22 & 0.47 & 0.47 & 0.23 & 0.23 & 0.08 & 0.08 & 1.0 & 1.0 & 0.001 & 0.003 & 0.006 & 0.004\end{array}$

\subsection{Residue analysis}

Residue analysis [19]-[20] is used to pair feedback signals with the control inputs in the damping control design. The matrix of transfer functions is:

$$
G(s)=C(s I-A)^{-1} B
$$

The transfer function between $j^{\text {th }}$ output and $k^{\text {th }}$ input of the plant $G(\mathrm{~s})$ is:

$$
G_{j k}(s)=\sum_{i=1}^{n} \frac{R_{i j k}}{\left(s-\lambda_{i}\right)}
$$

Where $R_{\mathrm{ijk}}$ is the residue associated with the $i^{\text {th }}$ mode, $j^{\text {th }}$ output and $k^{\text {th }}$ input:

$$
R_{i j k}=C_{j} \varphi_{i} \psi_{i} B_{k}
$$

$\lambda_{\mathrm{i}}$ is eigenvalue of the $i^{\text {th }}$ mode. Both $R_{\mathrm{ijk}}$ and $\lambda_{\mathrm{i}}$ can be real or complex values.

Fig. 4 shows the block diagram of a closed loop system with feedback between $y_{\mathrm{j}}$ and $u_{\mathrm{k}}$. $K H(\mathrm{~s})$ is the feedback loop that needs to be designed. $K$ is the gain of the feedback loop and $H(\mathrm{~s})$ is a compensator. The transfer function of the closed loop system of Fig. 4 can be expressed as

$$
G_{\text {close }}=\frac{G_{j k}(s)}{1+K H(s) G_{j k}(s)}
$$

The closed loop eigenvalues are the roots of

$$
1+K H(s) G_{j k}(s)=0
$$

Substitute (32) into (35) results

$$
1+K H(s)\left(\sum_{i=1}^{n} \frac{R_{i j k}}{s-\lambda_{i}}\right)=0
$$


The expression $K H(\mathrm{~s})$ is used to move a dominant eigenvalue $\lambda_{\mathrm{i}}$ left to a desired position $\lambda_{\mathrm{i} \text {,des }}$ while not significantly affecting position of the other eigenvalues. According to [19], if $R_{\mathrm{ijk}}$ associated with the dominant mode $\lambda_{\mathrm{i}}$ is much larger than $R_{\mathrm{mjk}}$ associated with other eigenvalues $\lambda_{\mathrm{m}}$, relationship between $\lambda_{\mathrm{i}, \text { des }}$ and the feedback loop is

$$
\Delta \lambda_{i}=\lambda_{i, d e s}-\lambda_{i}=-K H\left(\lambda_{i}\right) R_{i j k}
$$

In designing the feedback loop, the compensator $H(\mathrm{~s})$ is used to compensate the angle of the residue $R_{\mathrm{ijk}}$ so that $H\left(\lambda_{\mathrm{i}}\right) R_{\mathrm{ijk}}$ is a real number. Table 3 shows the calculated residues corresponding with the mode $-7.1+680 \mathrm{i}$ of the 4-port test system taking $V_{\mathrm{cd}}$ and $V_{\mathrm{cq}}$ as output while $M_{1 \mathrm{~d}}-M_{4 \mathrm{q}}$ as inputs. Table 3 shows that the magnitudes of the residues for each port are the same along the main diagonal or off diagonal, indicating that $M_{\mathrm{id}}-V_{\mathrm{cd}}$ and $M_{\mathrm{iq}}-V_{\mathrm{cq}}$ pairs, or alternatively, $M_{\mathrm{id}}-V_{\mathrm{cq}}, M_{\mathrm{iq}}-V_{\mathrm{cd}}$ pairs can be used to damp the oscillations. However diagonal pairing has large imaginary gain and it would require $90^{\circ}$ phase compensation. The off diagonal pairing is a better choice.

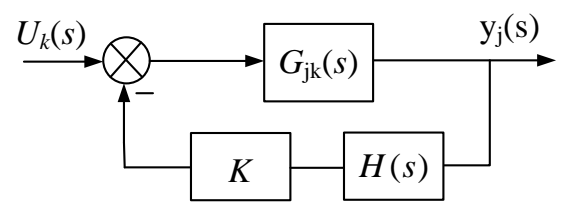

Fig. 4. Block diagram of closed-loop damping controller

\subsection{Damping controller using $V_{c q^{-}} M_{i d}$ and $V_{c d}-M_{\text {iq }}$ pairs}

According to Table 3, imaginary part of the residues for $V_{\mathrm{cq}}-M_{\mathrm{id}}$ and $V_{\mathrm{cd}}-M_{\mathrm{iq}}$ pairs are small and can be neglected. The $H(\mathrm{~s})$ can therefore be designed as a band-pass filter with a simple real gain and no phase compensation for the residues.

Table 3 Residues associated with $V_{\mathrm{cd}}$ and $V_{\mathrm{cq}}$

\begin{tabular}{lllllllll}
\hline \multicolumn{2}{c}{$M_{1 \mathrm{~d}}$} & $M_{1 \mathrm{q}}$ & $M_{2 \mathrm{~d}}$ & $M_{2 \mathrm{q}}$ & $M_{3 \mathrm{~d}}$ & $M_{3 \mathrm{q}}$ & $M_{4 \mathrm{~d}}$ & $M_{4 \mathrm{q}}$ \\
\hline$V_{\mathrm{cd}}$ & $-132-30137 \mathrm{i}$ & $30437-59 \mathrm{i}$ & $-556+127480 \mathrm{i}$ & $126480+648 \mathrm{i}$ & $-831-186280 \mathrm{i}$ & $188610+633 \mathrm{i}$ & $-467-90020 \mathrm{i}$ & $89547+1336 \mathrm{i}$ \\
\hline$V_{\mathrm{cq}}$ & $-30077+236 \mathrm{i}$ & $-164-30377 \mathrm{i}$ & $-127230+996 \mathrm{i}$ & $209-126240 \mathrm{i}$ & $-185910+1475 \mathrm{i}$ & $-21-18824 \mathrm{i}$ & $-89841+778 \mathrm{i}$ & $1023-89375 \mathrm{i}$ \\
\hline
\end{tabular}


The $\Delta \lambda_{\mathrm{i}}$ defined in (37) should be a negative value in order to shift eigenvalues to the left of the imaginary axis. Equation (37) further indicates that the gain $K$ will be of the same sign as the $R_{\mathrm{ijk}}$ on the condition that $R_{\mathrm{ijk}}$ is a real value.

Therefore, the gain of the damping loop for the $V_{\mathrm{cd}}-M_{\mathrm{iq}}$ pair is of positive value while the gain for the $V_{\mathrm{cq}}-M_{\mathrm{id}}$ is of negative sign.

The 'damping loop' of Fig. 2 shows the damping controller using $V_{\mathrm{cd}}-M_{\mathrm{iq}}$ and $V_{\mathrm{cq}}-M_{\mathrm{id}}$ pairs. The BPF stands for band pass filter with transfer function:

$$
H(s)=\frac{s / \omega_{b}}{1+2 \zeta_{b}\left(\frac{s}{\omega_{b}}\right)+\left(\frac{s}{\omega_{b}}\right)^{2}}
$$

For the test 4-port hub of Table 1 with all the four ports in operation, the characteristic frequency of the $\mathrm{BPF} \omega_{b}$ is adjusted to be $108 \mathrm{~Hz}$ according to the study of section 4.4. The damping ratio of the BPF is set to be $\xi_{\mathrm{b}}=0.707$. The BPF behaves close to a pure gain loop with no phase shift for the dominant modes of $V_{\mathrm{cd}}$ and $V_{\mathrm{cq}}$. Note that one damping loop can be used per port (say on $M_{\mathrm{d}}$ channel) but both control channels are used to enhance effectiveness and improve reliability.

\subsection{Robustness of the damping controller against topology changes}

Connecting or disconnecting ports will change the topology of the inner LCL circuit. The resonant frequency of the LCL DC hub will therefore also be changed. Table 4 shows resonant frequency of the test hub with different number of ports in operation. The '1234' means all the four ports are in operation. '234' means ports 2,3 and 4 are in operation.

Table 4 shows that the resonant frequency of the test hub changes notably after tripping a port. For each hub topology, it is necessary to adjust the characteristic frequency of the damping controller filter H(s). Since all the ports of the LCL dc hub are placed in a single or in several adjacent buildings [7]-[8], it is assumed that a supervisory controller will be sending the status of CBs to all ports, and local controllers can select the characteristic frequency of the local BPF filter. 
Table 4 Resonant frequency of the test hub with different number of ports in operation

\begin{tabular}{lccccccccccc}
\hline Operating ports & 1234 & 234 & 134 & 124 & 123 & 12 & 13 & 14 & 23 & 24 & 34 \\
& & & & & & & & & & & \\
\hline Resonant Frequency $(\mathrm{Hz})$ & 108 & 142 & 196 & 63 & 75 & 19 & 134 & 128 & 99 & 88 & 399 \\
\hline
\end{tabular}

\section{Simulation Verification}

\subsection{The test system}

Detailed model of the test 3-phase 4-port DC hub is developed on PSCAD/EMTDC with all switches adequately represented as on-off elements. The operating frequency is $1.25 \mathrm{kHz}$ and the modulation method as reported [7] is employed. Parameters of the test hub and initial power flow are listed in Table 1. The power loss of the AC inductor and the converter is modelled by a $1 \Omega$ resistor in series with each inductor $L_{\mathrm{i}}$. This parameter is not used in the linearized model and it tests the control performance in the presence of parameter uncertainties. The control parameters are listed in Table 5. Table 5 Control parameters of the test system

\begin{tabular}{lccccc}
\hline Parameters & $K_{\mathrm{pp}}$ & $K_{\mathrm{ip}}$ & $K_{\mathrm{pv}}$ & $K_{\mathrm{iv}}$ & $K_{\text {damp }}$ \\
\hline Value & 0.5 & 20 & 0.1 & 20 & 1.5 \\
& & & & & \\
\hline
\end{tabular}

\subsection{Startup and Step power response without power balance control}

Fig. 5 shows start up and system response without distributed power balance control. Initially, DC sides of each port are connected to DC voltage sources; AC circuit breaker of each port is connected to the common AC bus. Firing pulses for the IGBTs of all the ports are initially blocked, all the closed loop controls are disabled.

At 0.1 s, IGBTs of all the ports are enabled and $M_{\text {id }}$ of each port is increased from 0 to $M_{\text {idr }}$ during $0.1 \mathrm{~s}-0.4 \mathrm{~s}$. At $0.5 \mathrm{~s}$, ' $V_{\mathrm{cd}}$ loop' and 'Active power loop' of Fig. 2 are enabled; all the other loops remain disabled. Power order of each port is increased from 0 to the rated value of Table 1 during $0.5 \mathrm{~s}-0.6 \mathrm{~s}$. 
At 1.0s, the 'damping loop' is enabled. We can see from Fig. 5(a), (b) (d) during 0s-1.0s that the system can be started stably. ' $V_{\text {cq }}$ loop' remains being disabled throughout this test.

At $1.2 \mathrm{~s}$, power reference for port 2 is stepped from $1 \mathrm{pu}$ to $-1 \mathrm{pu}$. As a result, there is only port 4 injecting power to the hub, while all the other ports are required to absorb power at $1 \mathrm{pu}$. A similar power reference unbalance may happen in case of communication issues or local tripping of ports.

According to the analysis of section 3.2, while there are more total absorbing power orders than the total injecting power orders, a negative $V_{\mathrm{cq}}$ will result and this activates inherent power balancing mechanism in the hub.

Fig. 5(b) shows that the hub inherently decreases the power of absorbing ports and increases power of the injecting ports. Fig. 5(d) shows that $V_{\text {cq }}$ reaches as high as $-1.8 \mathrm{pu}$, which is significant over voltage to the hub. A hub without automatic power balance control cannot operate securely.
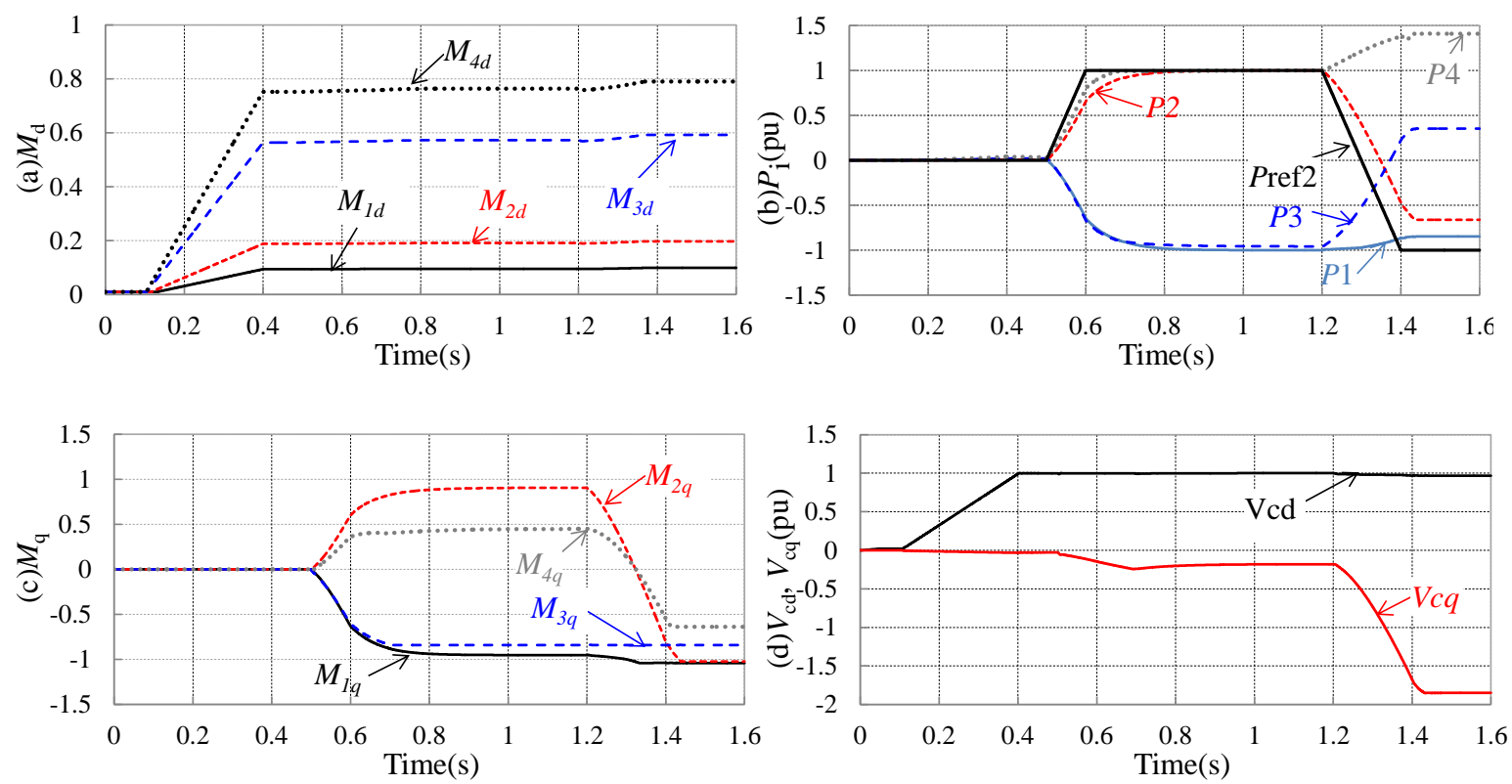

Fig. 5. Response to un-balanced step power order without power balancing control.

\subsection{Step power response with power balance control}

Fig. 6 shows system response with distributed power balance control. Extreme power unbalance is tested. DC power order of port 2 is stepped from $1 \mathrm{pu}$ to $-1 \mathrm{pu}$ at $1.0 \mathrm{~s}$ and restored back to $1 \mathrm{pu}$ at $1.4 \mathrm{~s}$. 
Power orders of other ports remain unchanged.

Fig. 6(a) shows that during 1.0s-1.4s each absorbing ports absorbs the same percentage of active power from the hub in steady state. DC power of each port is restored back to the values close to their reference values when the power orders revert back to the balanced condition after $1.4 \mathrm{~s}$.

It is also seen from Fig. 6(a) that before 1.0s the power of port 2 and port 4 are strictly regulated to their reference values while power of port 1 and port 3 are regulated to around $-0.97 \mathrm{pu}$. The small mismatch between $P_{1}, P_{3}$ and their reference values are because of the power loss of the hub. With the power loss taken into consideration, there are actually more absorbing power orders than the total injected power even with power orders of all the ports balanced.

Fig. 6(b) shows that there will be temporarily non-zero $V_{\mathrm{cq}}$ during the short period of power order changes. $V_{\mathrm{cq}}$ is well regulated to zero in steady state. The peak overvoltage magnitude $\left(\sqrt{V_{c d}^{2}+V_{c q}^{2}}\right)$ is around $12 \%$ which is acceptable. The LCL dc hub operates securely with the automatic power balancing controller.

Fig. 6(c) shows that during steady state and unbalanced power orders, $M_{1 q}^{\text {power }}$ and $M_{3 q}^{\text {power }}$ always hit the lower limit, $M_{2 q}^{\text {power }}$ hits the lower limit during unbalanced power orders. A positive $M_{\mathrm{cq}}$ is superimposed on each $M_{i q}^{\text {power }}$ to compensate the power loss during the balanced power orders and also to achieve power balance during un-balanced power orders.

Fig. 6(d) shows that each $M_{\text {id }}$ stays around rated value in steady state and during un-balanced power orders.
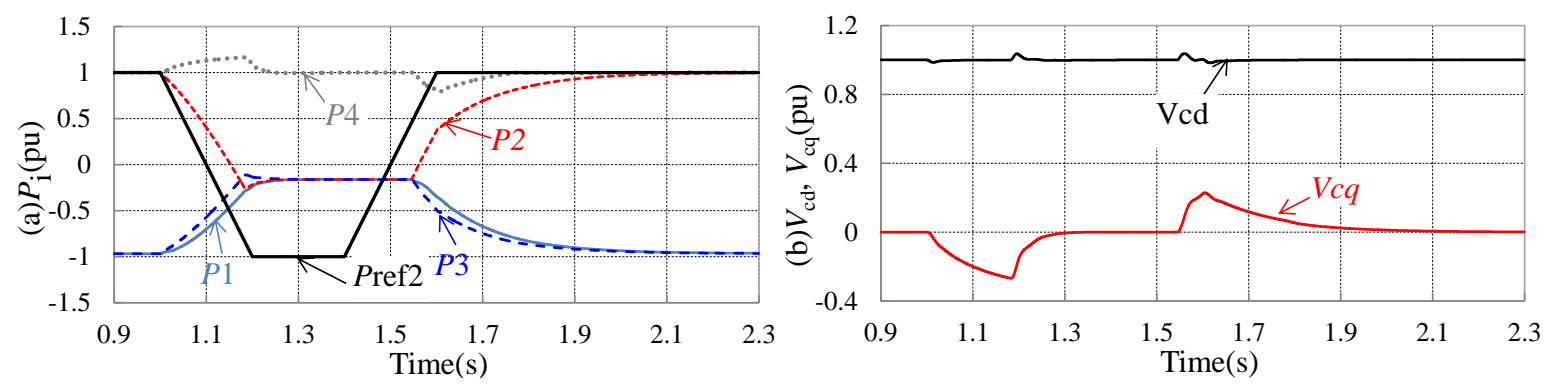

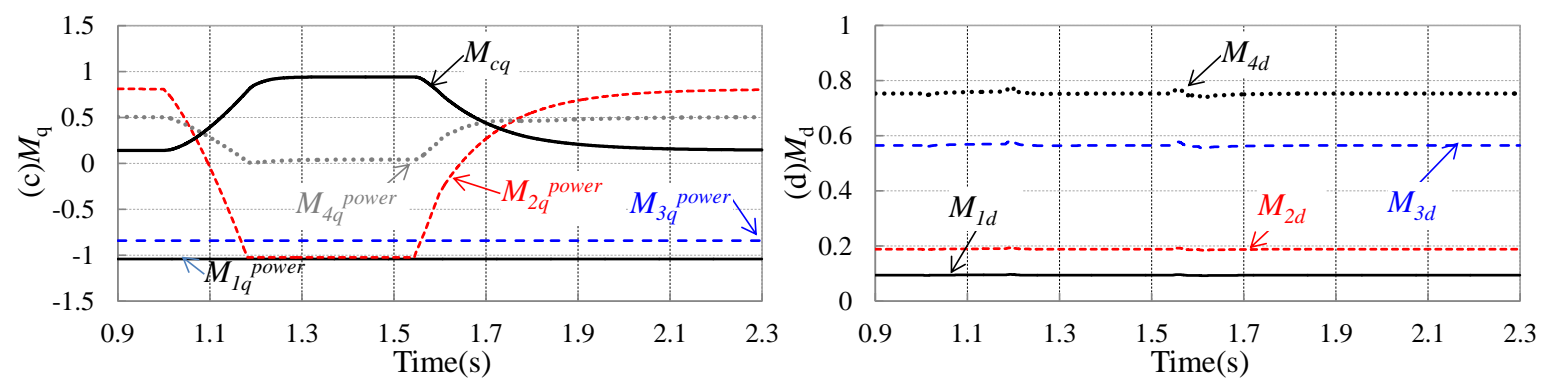

Fig. 6. Response to un-balanced step power order with power balancing control.

\subsection{Step power response with priority power balance control}

Fig. 7 shows system response with priority power balancing control. The applied power step is the same as Fig. 6, but the power sharing coefficient of port1 $\left(h_{1}\right)$ is set to zero, indicating port1 is a privileged port which cannot participate in the hub power balancing. In practice this can happen if the port is regulating DC line voltage (since remote terminal is wind farm that is uncontrollable current source) or the port is connected to a priority load with firm power requirements.

Fig. 7(a) shows that port1 is able to keep its active power at the power reference. Port2 and port3 reduce their absorbed active power to achieve power balance in the hub. Compared with Fig. 6(a) before 1.0s and after 2.1s, Fig. 7(a) also shows that power loss of the hub is compensated by the absorbing port3. Fig. 7(b) shows that $V_{\mathrm{cq}}$ is well regulated to zero in steady state.

The controller has been tested for wide range of other balanced and unbalanced power orders. It is always able to follow any balanced power order. For any unbalanced power order, $V_{\mathrm{cd}}$ and $V_{\mathrm{cq}}$ are well regulated and port powers are moderated according to coefficients $h_{\mathrm{i}}$.
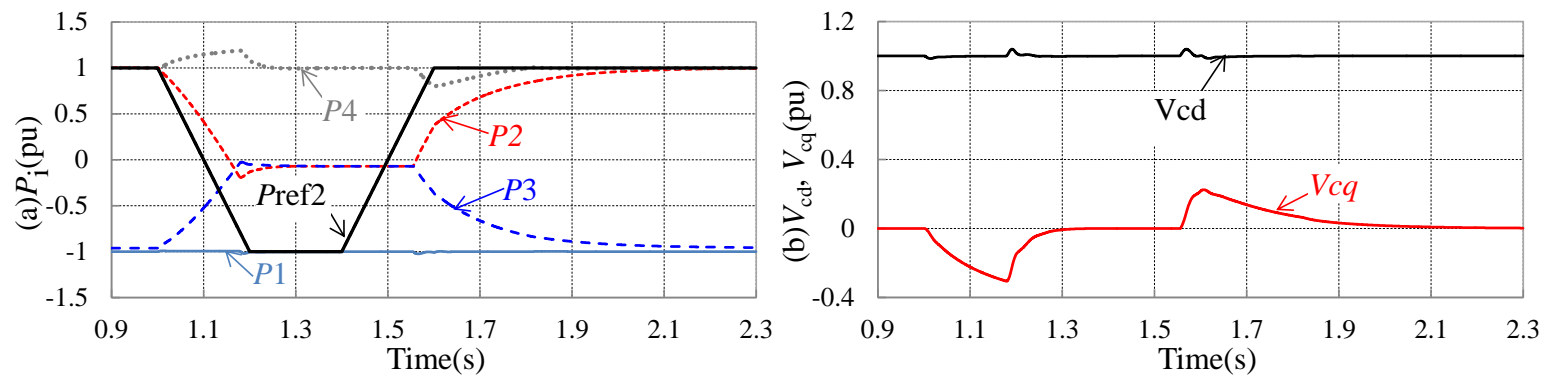

Fig. 7. Response to un-balanced step power order with priority power balance control. 


\subsection{Verification of the damping controller}

Fig. 8 shows system response with and without the damping controller. A permanent dc fault is applied at the dc terminals of port 4 at $1 \mathrm{~s}$ and port 4 is tripped from the hub by its $\mathrm{AC}$ circuit breaker at 1.1s. The 'NoDamp' and 'Damp' respectively stands for topology without and with damping controller. Fig. 8 shows that the damping controller is able to significantly improve the damping ratio and also reduce the peak value of transient response.
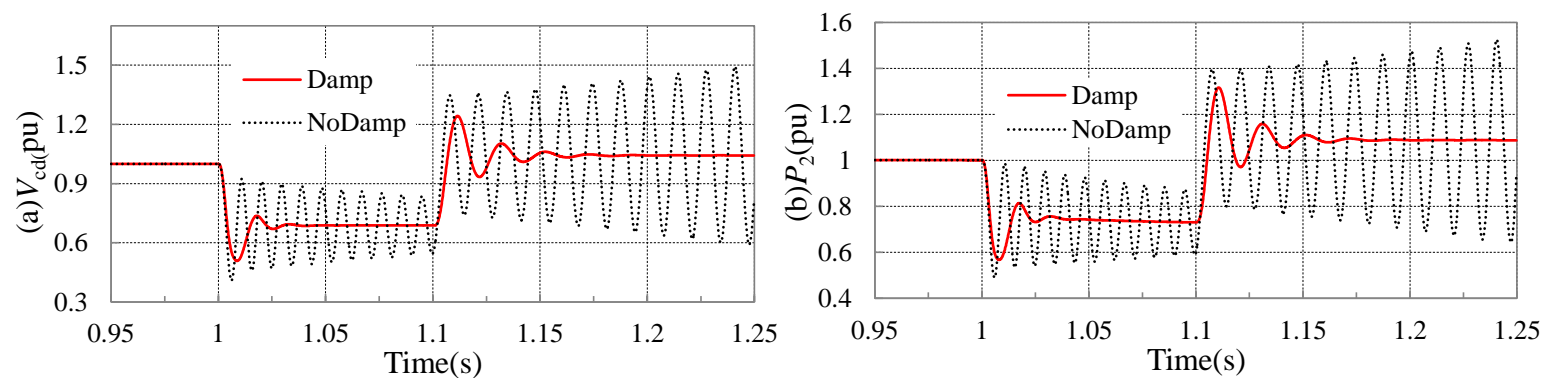

Fig. 8. Comparisons of the damping controllers.

\subsection{Effect of topology change on the damping controller}

Fig. 9 shows the impact of topology change on the damping controller. A permanent dc fault is applied at dc side of port 4 at 1.0s and port4 is tripped from the hub by its AC circuit breaker at 1.1s. Another temporary dc fault with duration of $0.05 \mathrm{~s}$ is then applied at the dc side of port 2 at $1.3 \mathrm{~s}$ to test performance of the damping controller when topology of the hub is different.

The solid line of Fig. 9(a) shows system response with the characteristic frequency of BPF kept at $108 \mathrm{~Hz}$, which is the dominant oscillatory mode of the 4-port hub while the dashed line of Fig. 9(a) shows the result with the BPF kept at $75 \mathrm{~Hz}$, which is the dominant oscillating mode for a hub with port1-port3 in operation.

We can see from Fig. 9(a) that the BPF tuned at $75 \mathrm{~Hz}$ offers better damping for the remaining 3-port hub during the fault at port4 and after tripping of port4. However, the BPF originally tuned for the 4-port hub still offers acceptable damping effect during the topology change of the hub. 
Fig. 9(b) shows active powers of the four ports. Characteristic frequency of the BPF is kept at $108 \mathrm{~Hz}$ during the tripping of port4 as it is not a common practice to adjust the control parameters when the disturbance is in process. The characteristic frequency is adjusted to $75 \mathrm{~Hz}$ at $1.2 \mathrm{~s}$ after port4 has been tripped. We can see from the curves of Fig. 9(b) after 1.3s that the re-tuned BPF offers sufficient damping for the remaining 3-port hub.

In practical operation, a look-up table according to Table 4 can be kept in the supervisory control of the hub. Characteristic frequency of all the ports will be adjusted according to Table 4 depending on the ON/OFF state of the AC circuit breaker of each port. We assume that characteristics of the BPF of all the ports will be re-tuned $100 \mathrm{~ms}$ after detecting topology change of the hub. The $100 \mathrm{~ms}$ delay after tripping a port is introduced to avoid further disturbance of the system because of re-tuning the BPF parameter.

Fig. 9 also illustrates the effectiveness of power balancing control under DC faults and port tripping.
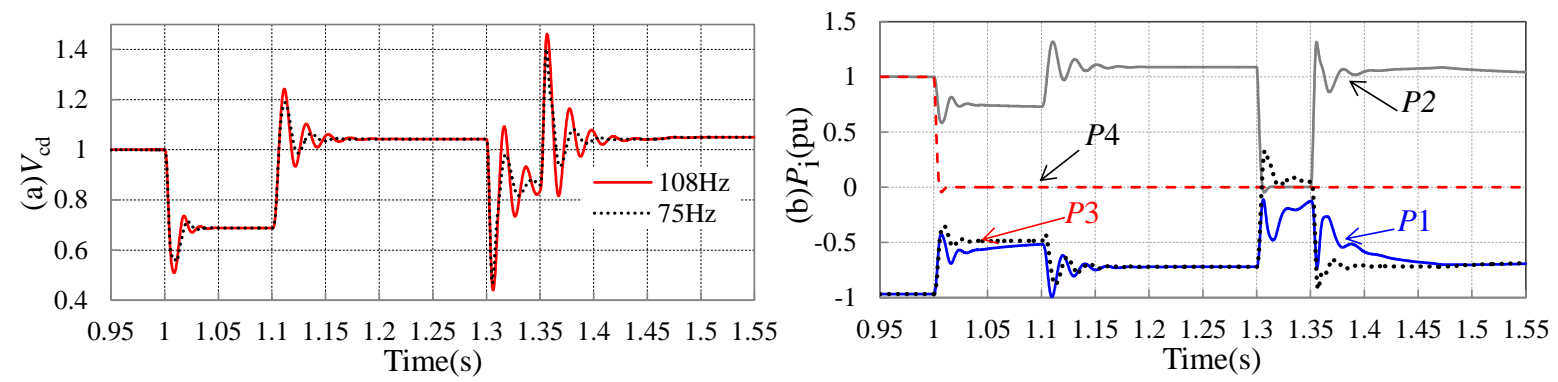

Fig. 9. Impact of topology change on the damping controller.

\section{Conclusion}

Distributed power balance and damping controller for high power multiport LCL DC hub is proposed in this paper. The distributed power balance control enables secure operation of the LCL DC hub for arbitrary power reference at individual ports and for disturbances including topology change. Each port is able to independently control its active power but port power is moderated 
depending on the power balance in the hub. It is possible to adjust the contribution of each port in the hub power balancing and a zero contribution is also feasible. Damping controllers are designed to suppress the dominant oscillatory modes caused by the LCL circuit in the hub. Eigenvalue and residue analysis shows that $V_{\mathrm{cd}}-M_{\mathrm{iq}}$ and $V_{\mathrm{cq}}-M_{\mathrm{id}}$ signal pairings are best for damping oscillations. Simulations on PSCAD/EMTDC confirm the effectiveness of the designed controllers.

\section{Acknowledgements}

This project is funded by European Research Council in FP7; grant no 259328, 2010 and EPSRC grant no EP/K006428/1, 2013.

\section{References}

[1] D. Van Hertem, M. Ghandhari, Multi-terminal VSC HVDC for the European Supergrid: obstacles, Renewable and Sustainable Energy Reviews. 14(9) (2010) 3156-3163.

[2] M. Barnes, A. Beddard, Voltage source converter HVDC links - the state of the art and issues going forward, Energy Procedia. 24(2012) 108-122.

[3] X. Zhao, K. Li, Droop setting design for multi-terminal HVDC grids considering voltage deviation impacts, Electric Power Systems Research 123 (2015) 67-75.

[4] M. Aragüés-Peñalba, A. Egea-Àlvarez, S. Arellano, O. Gomis-Bellmunt, Droop control for loss minimization in HVDC multi-terminal transmission systems for large offshore wind farms, Electric Power Systems Research, 112(2014) 48-55.

[5] O. Gomis-Bellmunt, J. Liang, J. Ekanayake, R. King, N. Jenkins, Topologies of multiterminal HVDC-VSC transmission for large offshore wind farms, Electric Power Systems Research, 81 (2011) 271-281.

[6] C. Barker, C. Davidson, D. Trainer, R. Whitehouse, Requirements of DC-DC converters to facilitate large DC Grids, in: CIGRE Session, 2012. 
[7] D. Jovcic, W. Lin, Multiport high power LCL DC hub for use in DC Transmission Grids, IEEE Trans. Power Del., 29(2) (2014) 760-768.

[8] W. Lin, D. Jovcic, Reconfigurable multiphase multi GW LCL DC hub with high security and redundancy, Electric Power Systems Research, 110(2014) 104-112.

[9] S. I. Nanou, G. N. Patsakis, S. A. Papathanassiou, Assessment of communication-independent rid code compatibility solutions for VSC-HVDC connected offshore wind farms, Electric Power Systems Research, 121 (2015) 38-51.

[10]J. Zhu, C. D. Booth, G. P. Adam, A. J. Roscoe, Coordinated direct current matching control strategy for multi-terminal DC transmission systems with integrate wind farms, Electric Power Systems Research, 124 (2015) 55-64.

[11]T. K. Vrana, J. Beerten, R. Belmans, O. Fosso, A classification of DC node voltage control methods for HVDC grids, Electric Power Systems Research, 103 (2013) 137-144.

[12]A. Egea-Alvarez, J. Beerten, D. V. Hertem, O. Gomis-Bellmunt, Hierarchical power control of multiterminal HVDC grids, Electric Power Systems Research, 121 (2015) 207-215.

[13]K. Rouzbehi, A. Miranian, A. Luna, P. Rodriguez, DC voltage control and power sharing in multiterminal DC grids based on optimal power flow and voltage-droop strategy, IEEE Journal of Emerging and Selected Topics in Power Electronics, 2(4) (2014) 1171-1180.

[14]L. Harnefors, N. Johansson, L. Zhang, B. Berggren, Interarea oscillation damping using active-power modulation of multiterminal HVDC transmissions, IEEE Trans. Power Syst., 29(5)(2014) 2529-2538.

[15]L. Harnefors, N. Johansson, L. Zhang, B. Berggren, Interarea oscillation damping using active-power modulation of multiterminal HVDC transmissions, IEEE Trans. Power Syst., 29(5)(2014) 2529-2538. 
[16]S. M. Fazeli, D. Jovcic, W. Lin, Distributed control for LCL DC hub enabling reliable energy transfer in future DC grids, in: Proc. IET ACDC 2015, 2015.

[17]Kundur, P. Power System Stability and Control, McGraw Hill, 1994.

[18]S. Skogestad and I. Postlethwaite, Multi Variable Feedback Control Analysis and Design, John Wiley \& Sons, 2004.

[19]N. Yang, Q. Liu, J. D. McCalley, TCSC controller design for damping interarea oscillations, IEEE Trans. Power Syst., 13(4) (1998) 1304-1310.

[20]Y. Li, C. Rehtanz, S. Rüberg, L. Luo, Y. Cao, Assessment and choice of input signals for multiple HVDC and FACTS wide-area damping controllers, IEEE Trans. Power Syst., 27(4) (2012) 1969-1977.

[21]D Jovcic and K Ahmed, High Voltage Direct Current Transmission: Converters Systems and DC Grids, Wiley, 2015, 